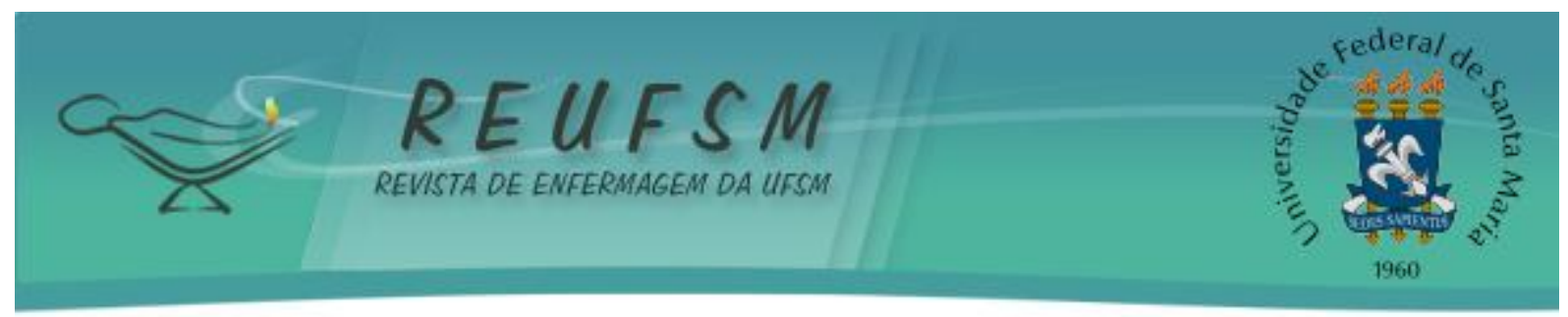

ARTIGO ORIGINAL

\title{
PERCEPÇÕES CULTURAIS DE ACADÊMICOS E ENFERMEIROS SOBRE A SISTEMATIZAÇÃO DA ASSISTÊNCIA DE ENFERMAGEM
}

\author{
CULTURAL PERCEPTIONS OF STUDENTSAND NURSES ABOUT THE NURSING CARE \\ SYSTEMATIZATION
}

\section{PERCEPCIONES CULTURALES DE ESTUDIANTES Y ENFERMEROS SOBRE LA SISTEMATIZACIÓN DE LA ASISTENCIA DE ENFERMERÍA}

\author{
Vander Monteiro da Conceição ${ }^{1}$ \\ Jeferson Santos Araujo ${ }^{2}$ \\ Rafaela Azevedo Abrantes de Oliveira ${ }^{3}$ \\ Márcia Maria Fontão Zago ${ }^{4}$ \\ Ralrizônia Fernandes Souza ${ }^{5}$ \\ Mary Elizabeth de Santana ${ }^{6}$ \\ Maria Odeise da Paixão Monteiro ${ }^{7}$ \\ Aline Bento Neves 8 \\ Viviane de Oliveira Gomes ${ }^{9}$ \\ Michelle Pena Nery Luz ${ }^{10}$
}

Doi: $10.5902 / 2179769211234$

RESUMO: Objetivo: identificar as percepções de acadêmicos e enfermeiros sobre a sistematização da assistência de enfermagem em relação ao ensino e aplicabilidade. Método: estudo de caráter descritivo e delineamento de levantamento, com foco em aspectos subjetivos a partir da perspectiva cultural. Entrevistou-se 28 acadêmicos de enfermagem e 20 enfermeiros. Os relatos foram analisados segundo a análise temática dedutiva. Resultados: obteve-se as seguintes unidades temáticas: "A SAE como instrumento para o cuidado de qualidade", "O ensino da SAE e o cuidado prestado" e "A aplicabilidade da SAE". Conclusões: o ensino e a aplicação da SAE, no contexto estudado, ainda não conseguem produzir resultados almejados, tais como a melhora no

\footnotetext{
${ }^{1}$ Enfermeiro, Doutorando em Ciências pelo Programa de Pós-Graduação em Enfermagem Fundamental, Escola de Enfermagem de Ribeirão Preto - USP, Ribeirão Preto, São Paulo, Brasil. Bolsista de Doutorado FAPESP. Email: vandermonteiro@usp.br;

${ }^{2}$ Enfermeiro, Doutorando em Ciências pelo Programa de Pós-Graduação em Enfermagem Fundamental, Escola de Enfermagem de Ribeirão Preto - USP, Ribeirão Preto, São Paulo, Brasil. Bolsista de Doutorado CNPq. E-mail: jefaraujo@usp.br;

${ }^{3}$ Enfermeira, Mestranda em Ciências pelo Programa de Pós-Graduação em Enfermagem Fundamental, Escola de Enfermagem de Ribeirão Preto - USP, Ribeirão Preto, São Paulo, Brasil. Bolsista de Mestrado FAPESP. E-mail: rafaelazevedo@usp.br;

${ }^{4}$ Enfermeira, Doutora em Enfermagem, Professor Sênior da Escola de Enfermagem de Ribeirão Preto - USP, Ribeirão Preto, São Paulo, Brasil. Bolsista de Produtividade em Pesquisa CNPq. E-mail: mmfzago@eerp.usp.br;

${ }^{5}$ Enfermeira, Mestranda em Enfermagem pelo Programa de Pós-Graduação em Enfermagem da Escola de Enfermagem Magalhães Barata - UEPA, Belém, Pará, Brasil. E-mail: rjna2005@hotmail.com;

${ }^{6}$ Enfermeira, Doutora em Enfermagem, Professor Titular da Enfermagem Magalhães Barata - UEPA, Belém, Pará, Brasil. E-mail: betemary@terra.com.br;

7 Enfermeira, Especialista em Enfermagem Obstétrica e Neonatal, Hospital Maternidade de Tomé-Açu; ToméAçu, Pará, Brasil. E-mail: odeisepaixao@hotmail.com;

${ }^{8}$ Enfermeira, Especialista em Enfermagem em Nefrologia, Hospital Adventista de Belém, Belém, Pará, Brasil. E-mail: alinebentoneves@hotmail.com;

9 Enfermeira, Especialista em Enfermagem Obstétrica e Neonatal, Prefeitura Municipal de Capanema, Capanema, Pará, Brasil. E-mail: vivi---gomes@hotmail.com;

10 Enfermeira, Especialista em Nefrologia e Administração Hospitalar, Professora da Escola Superior da Amazônia, Belém, Pará, Brasil. E-mail: michellipena@yahoo.com.br.
} 


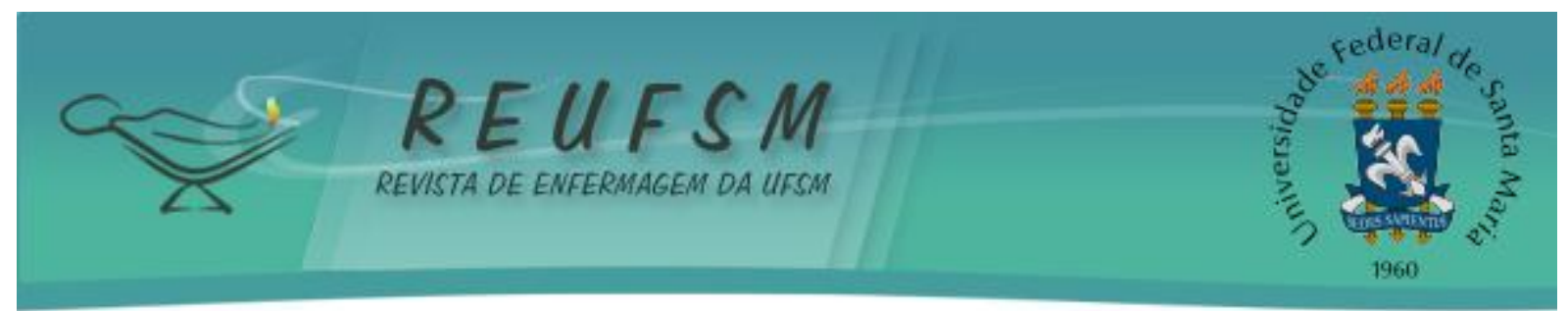

dimensionamento de enfermagem e união entre a equipe, que facilitaria a execução da SAE na clínica. A participação efetiva dos docentes com domínio teórico e instrumental facilitaria a construção e aplicação dos processos de enfermagem.

Descritores: Cuidados de enfermagem; Processos de enfermagem; Educação em enfermagem.

ABSTRACT: Aim: to identify the perceptions of students and nurses on the nursing care systematization in relation to teaching and applicability. Method: study design and descriptive survey, focusing on subjective from the cultural perspective aspects. We interviewed 28 nursing students and 20 nurses. The reports were analyzed according to deductive thematic analysis. Results: the following thematic units were obtained: "The NCS as a tool for quality care", "The teaching of NCS and the care provided" and "The applicability of NCS". Conclusions: the teaching and application of NCS in the context studied, yet fail to produce desired results, such as improvement in scaling nursing and unity among the team, which would facilitate the implementation of NCS in the clinic. The effective participation of teachers with theoretical and working knowledge facilitates the construction and application of the nursing process.

Descriptors: Nursing care; Nursing process; Education, nursing.

RESUMEN: Objetivo: identificar las percepciones de estudiantes y enfermeros en la sistematización de la asistencia de enfermería en relación con la enseñanza y su aplicabilidad. Método: estudio descriptivo, centrándose en los aspectos subjetivos desde la perspectiva cultural. Entrevistamos a 28 estudiantes de enfermería y 20 enfermeros. Los informes fueron analizados según el análisis temático deductivo. Resultados: Surgieron las siguientes unidades temáticas: "El SAE como instrumento para la atención de calidad", "La enseñanza de la SAE y la atención prestada" y "La aplicabilidad del SAE". Conclusiones: la enseñanza y la aplicación de la SAE en el contexto estudiado, no producen los resultados deseados, tales como la mejora en la dimensión de la enfermería y la unidad entre el equipo, lo que facilitaría la implementación del SAE en la clínica. La participación efectiva de los docentes con el conocimiento teórico e instrumental facilitaría la construcción y aplicación de los procesos de enfermería.

Descriptores: Atención de enfermería; Procesos de enfermería; Educación en enfermería.

\section{INTRODUÇÃO}

A Sistematização da Assistência de Enfermagem (SAE) presume a organização em um sistema que por sua vez requer um conjunto de elementos, dinamicamente inter-relacionados. Enquanto o Processo de Enfermagem (PE) é uma sequência de etapas privativas de obtenção de informações multidimensionais sobre o estado de saúde, identificação das necessidades humanas básicas afetadas que requerem intervenções de enfermagem, com objetivo de prestar atendimento profissional ao cliente, seja ele indivíduo, família ou comunidade, de maneira a considerar suas singularidades e de modo estendido. ${ }^{1}$

A SAE é hoje reconhecida como um importante instrumento metodológico capaz de nortear o cuidado prestado pelo enfermeiro e sua equipe, devendo a mesma ser registrada formalmente de acordo com o Conselho Federal de Enfermagem (COFEN) pela resolução $n^{\circ} 358 / 2009 .^{2}$

A execução do SAE enfrenta desafios em relação ao seu ensino, pois se necessita de professores capacitados e campos de prática que valorizem a ferramenta durante a assistência ao paciente. ${ }^{3}$ 


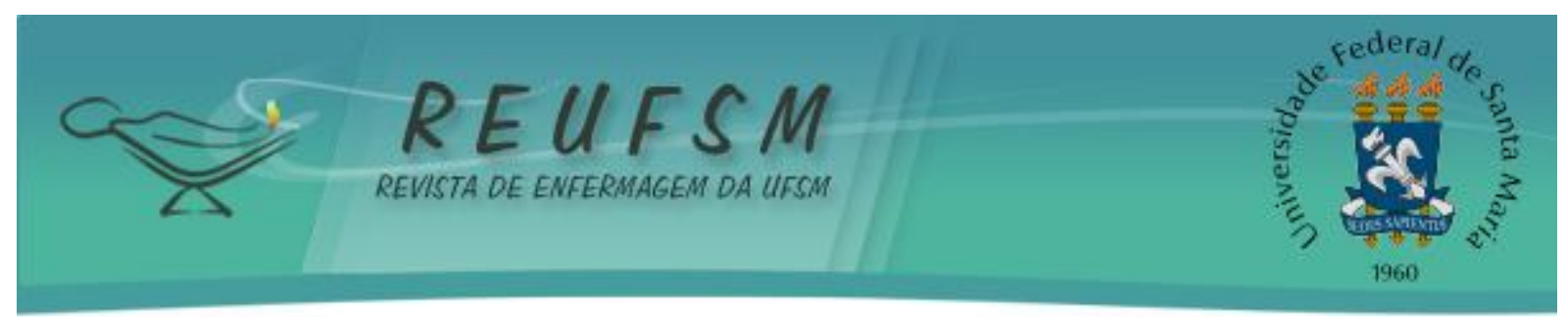

È neste cenário que surgem as preocupações com os dois sujeitos deste estudo, o acadêmico de enfermagem e o enfermeiro, para conhecer a percepção destes sobre a SAE e posteriormente, o desenvolvimento de estratégias para a resolução de possíveis problemas e o aperfeiçoamento do cuidar a ser prestado. Então, elaborou-se a seguinte questão norteadora: Quais as percepções dos acadêmicos e enfermeiros sobre a sistematização da assistência de enfermagem em relação ao seu ensino e aplicabilidade?

Para o desenvolvimento deste estudo objetivou-se identificar as percepções de acadêmicos e enfermeiros sobre a sistematização da assistência de enfermagem em relação ao seu ensino e aplicabilidade.

\section{MÉTODO}

Estudo descritivo, onde se buscou ressaltar os aspectos culturais presentes nos relatos dos sujeitos em estudo, partindo do conceito de cultura ${ }^{4}$, que envolve as dimensões que influenciam diretamente as ideias e os comportamentos dos indivíduos em relação à aplicação do processo de enfermagem. Este artigo ressaltou o cuidado formal a um paciente, pois trata da cultura de formação do enfermeiro para a prática da SAE, visualizado em dois contextos, o acadêmico e o profissional.

$\mathrm{O}$ estudo envolveu dados de dois projetos. O primeiro projeto (PP) “A percepção do acadêmico de enfermagem sobre a sistematização da assistência de enfermagem" foi desenvolvido na Faculdade de Enfermagem (FAENF) da Universidade Federal do Pará (UFPA), mediante a autorização da diretora do curso de Licenciatura e Bacharelado em Enfermagem. E a posterior aprovação do Comitê de Ética em Pesquisa com Seres Humanos da Universidade do Estado do Pará (protocolo $n^{\circ}$ 0036.073.321-09). A FAENF/UFPA foi escolhida como local de estudo por ser a instituição onde parte dos pesquisadores desenvolvem suas atividades de ensino.

0 PP teve 28 participantes, sendo eles, acadêmicos de enfermagem da referida instituição de ensino. Os mesmos concordaram com as informações contidas no Termo de Consentimento Livre e Esclarecidas (TCLE) e, por meio de sua assinatura, legalizaram sua inclusão no estudo. Foram pré-estabelecidos critérios de inclusão: ser maior de 18 anos e ter cursado 50\% do curso de graduação (4 semestres) devido a vivência com a SAE na teoria e na prática.

A coleta de dados ocorreu por meio de entrevista individual, no período de janeiro a junho de 2010. Os alunos foram abordados em sala de aula nos intervalos das atividades curriculares. Como instrumento de coleta de dados utilizou-se um questionário sociodemográfico e um roteiro de entrevista contemplando os seguintes aspectos: (1) Percepções sobre a SAE; (2) Percepções sobre o ensino da SAE; e (3) Percepções sobre a aplicabilidade da SAE. Ressalta-se que as entrevistas foram realizadas aproximadamente em uma hora.

O segundo projeto (SP) "As representações de enfermeiros sobre a sistematização da assistência de enfermagem e suas implicações para o cuidado", foi desenvolvido no Hospital Ophir Loyola (HOL), mediante autorização da Gerência de Enfermagem e posterior aprovação do Comitê de Ética em Pesquisa com Seres Humanos da Fundação Hospital das Clínicas Gaspar Viana (protocolo $\mathrm{n}^{\circ}$ 235/2010). O HOL foi selecionado para 0 desenvolvimento do SP por ser uma instituição pública de saúde de alta complexidade, com um número de enfermeiros elevados, além de ser um dos hospitais que têm a SAE como rotina nas atividades de enfermagem e ser campo de prática dos alunos da FAENF/UFPA.

Aceitaram participar do SP 20 enfermeiros da referida instituição de saúde, que concordaram com as informações contidas no TCLE e garantiram sua participação por meio da assinatura do mesmo. Como critérios de inclusão ficou estabelecido: ser maior de 18 anos, trabalhar na assistência direta aos usuários do presente serviço de saúde e atuar na 


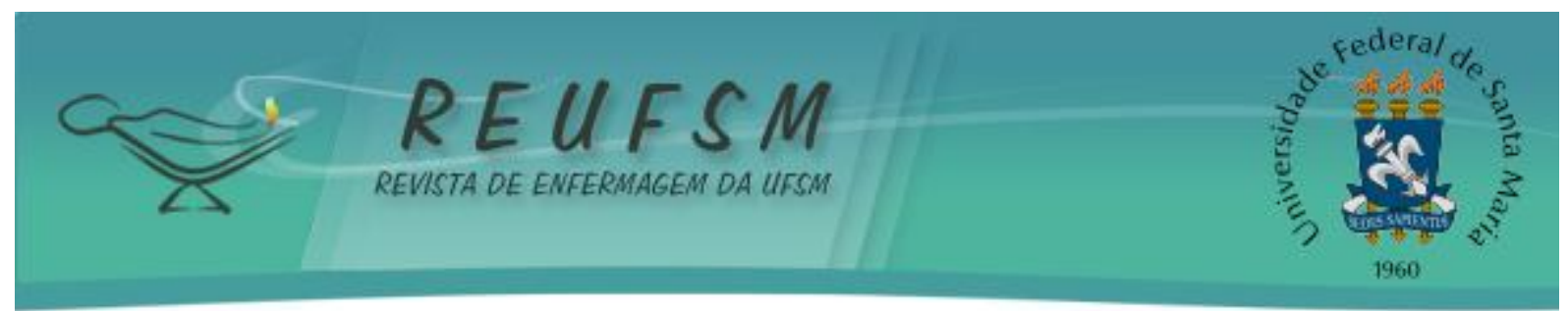

instituição como enfermeiro há mais de um ano, período pressuposto de que o sujeito teve possibilidade de se adaptar à prática do processo de enfermagem.

No SP a coleta de dados ocorreu por meio da entrevista individual, no período de novembro de 2010 a fevereiro de 2011. Os enfermeiros foram abordados nos intervalos de suas atividades laborais. Os instrumentos de coleta foram um questionário sociodemográfico e um roteiro de entrevista contemplando os seguintes aspectos: (1) Percepções sobre o cuidado de enfermagem; (2) Percepções sobre a SAE; e (3) Percepções sobre a aplicabilidade da SAE. As entrevistas duraram aproximadamente uma hora.

Para ambos os projetos os dados sociodemográficos foram analisados segundo a estatística descritiva. Os relatos obtidos foram transcritos em texto e analisados segundo a análise temática dedutiva, sendo classificados segundo sua frequência e importância para o estudo, em categorias comuns, que foram transformadas em unidades temáticas. ${ }^{5}$

Este estudo está de acordo com as normas da resolução 196/96 do Conselho Nacional de Saúde que dispõe sobre "Diretrizes e Normas Regulamentadoras de Pesquisas Envolvendo Seres Humanos". 6 Os sujeitos dos estudos foram identificados alfanumericamente quando citados em suas falas no artigo, sendo que ao se tratar de Acadêmicos de Enfermagem foram denominados de Acadêmico 1, Acadêmico 2,... Acadêmico $\mathrm{n}$, desta mesma forma os Enfermeiros denominados de Enfermeiro 1, Enfermeiro 2,... Enfermeiro $\mathrm{n}$.

\section{RESULTADOS}

\section{Contextualização dos dados sociodemográficos}

A partir dos dados sociodemográficos elaborou-se a Tabela 1, tendo como variáveis destacadas a faixa etária, gênero, semestre em curso (para acadêmicos) e tempo de formação (para enfermeiros).

Tabela 1- Distribuição dos sujeitos segundo a faixa etária, gênero, semestre em curso e tempo de formação, Belém, Pará, Brasil, 2012.

\begin{tabular}{|c|c|c|c|c|c|c|c|c|c|c|}
\hline Sujeitos & Idade & $\mathrm{F}$ & $\%$ & Gênero & $\mathrm{F}$ & $\%$ & & $T$ & $\mathrm{~F}$ & $\%$ \\
\hline \multirow{5}{*}{ 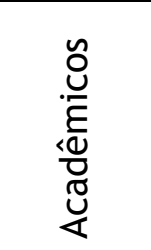 } & $19-25$ & 15 & 53,6 & $M$ & 4 & 14,3 & \multirow{5}{*}{ 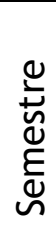 } & $5^{\circ}$ & 4 & 14 \\
\hline & $26-30$ & 8 & 28,6 & $\mathrm{~F}$ & 24 & 85,7 & & $6^{\circ}$ & 5 & 18 \\
\hline & $31-40$ & 5 & 17,8 & TP & 28 & 100 & & $7^{\circ}$ & 15 & 54 \\
\hline & \multirow[t]{2}{*}{ TP } & \multirow[t]{2}{*}{28} & \multirow[t]{2}{*}{100} & & & & & $8^{\circ}$ & 4 & 14 \\
\hline & & & & & & & & TP & 28 & 100 \\
\hline \multirow{8}{*}{ 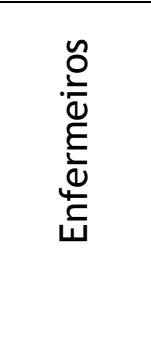 } & $23-29$ & 5 & 25 & M & 3 & 15 & \multirow{8}{*}{ 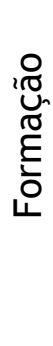 } & $2-6$ anos & 3 & 15 \\
\hline & $30-39$ & 9 & 45 & $\mathrm{~F}$ & 17 & 85 & & $7-11$ anos & 4 & 20 \\
\hline & $42-49$ & 3 & 15 & TP & 20 & 100 & & $12-16$ anos & 6 & 30 \\
\hline & $50-55$ & 3 & 15 & TG & 48 & - & & $17-21$ anos & 4 & 20 \\
\hline & TP & 20 & 100 & & & & & $22-26$ anos & 2 & 10 \\
\hline & TG & 48 & 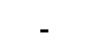 & & & & & $27-30$ anos & 1 & 5 \\
\hline & & & & & & & & TP & 20 & 100 \\
\hline & & & & & & & & TG & 48 & \\
\hline
\end{tabular}

Legenda: $M=$ Masculino; $F=$ Feminino; $T P=$ Total parcial; $T G=$ Total geral; $T=$ tempo; $F=$ frequência. Fonte: Pesquisa de campo, 2012.

Pela tabela 1 conclui-se que a maioria dos acadêmicos de enfermagem estava na faixa etária de 19 a 25 anos de idade $(53,6 \%$ ) e em menor número na faixa de 31 a 40 anos 


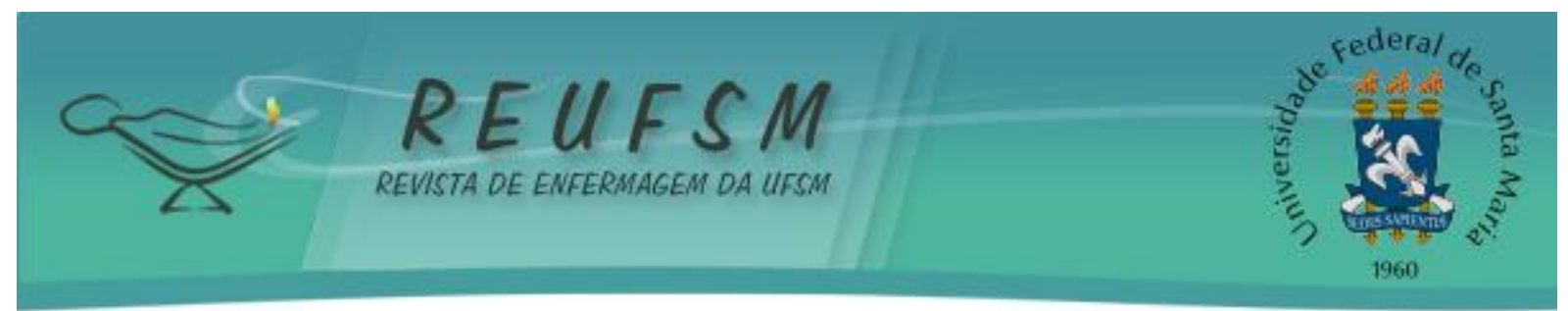

$(17,8 \%)$. A faixa etária dos enfermeiros que participaram do estudo demonstrou a prevalência de sujeitos entre 30 a 39 anos (45\%) e em pequena porcentagem, profissionais com 50 a 55 anos (15\%). Os sujeitos entrevistados eram na maioria do gênero feminino, $85,7 \%$ para acadêmicos de enfermagem e $85 \%$ para enfermeiros, mostrando ainda a prevalência do gênero feminino nesse contexto.

Os dados apresentados na Tabela 1 demonstram que a prevalência dos entrevistados graduandos foi do sétimo semestre (54\%), fator de grande relevância ao estudo, pois quanto mais próximo do término do curso, maior foi o contato do aluno com a $\mathrm{SAE}$, tanto na teoria como na prática; porém, não invalida e nem torna irrelevante as informações coletadas pelos grupos anteriores ao sétimo semestre.

O tempo de formação foi uma das variáveis coletadas para avaliação, pois como um dos questionamentos para os enfermeiros foi o cuidado de enfermagem, logo se teria fragmentos de momentos históricos diferentes nos relatos, uma vez que a formação pode inferir diretamente na visão de cuidado dos sujeitos, permitindo também conhecer alguns aspectos do ensino em enfermagem.

Desta forma, percebe-se que o tempo maior de formação é de 28 anos (5\%), todavia a maior frequência esteve presente no grupo de sujeitos formados no intervalo de 12 a 16 anos (30\%), e os sujeitos com o tempo de formação menor, de dois a seis anos, corresponderam ao total de $15 \%$ dos enfermeiros entrevistados. seguintes:

Ao analisar as questões subjetivas emergiram três unidades temáticas que são as

\section{A SAE como instrumento para o cuidado de qualidade}

Nesta primeira unidade temática apresentam-se os aspectos comuns sobre a SAE entre os sujeitos. Um aspecto inicial evidenciado foi a ideia de "autonomia profissional".

A SAE é um instrumento profissional do enfermeiro, que direciona sua prática e pode fornecer autonomia profissional e realizar a proposta de promover, manter ou restaurar o nível de saúde do paciente, como também documentar sua prática profissional, tendo em vista a avaliação da qualidade da assistência oferecida. (Acadêmico 2)

A SAE é uma atividade privativa do enfermeiro, que através de um método e estratégia de trabalho científico realiza a identificação das situações de saúde, subsidiando a prescrição e a implementação da assistência de enfermagem, que possam contribuir para a promoção, prevenção, recuperação e reabilitação em saúde do individuo, família e comunidade. (Acadêmico 5)

É através da SAE que o enfermeiro assume o seu específico clinicar; é o instrumento de trabalho que dá subsídio e respalda cientificamente a assistência de enfermagem. (Enfermeiro 19)

Os sujeitos também perceberam que a SAE é uma importante ferramenta de trabalho para a Enfermagem, porém os acadêmicos caracterizaram do ponto de vista teórico, algo condizente já que estão em processo de formação. No entanto, os enfermeiros realizaram a associação da SAE ao seu contexto de trabalho, apresentados a seguir: 


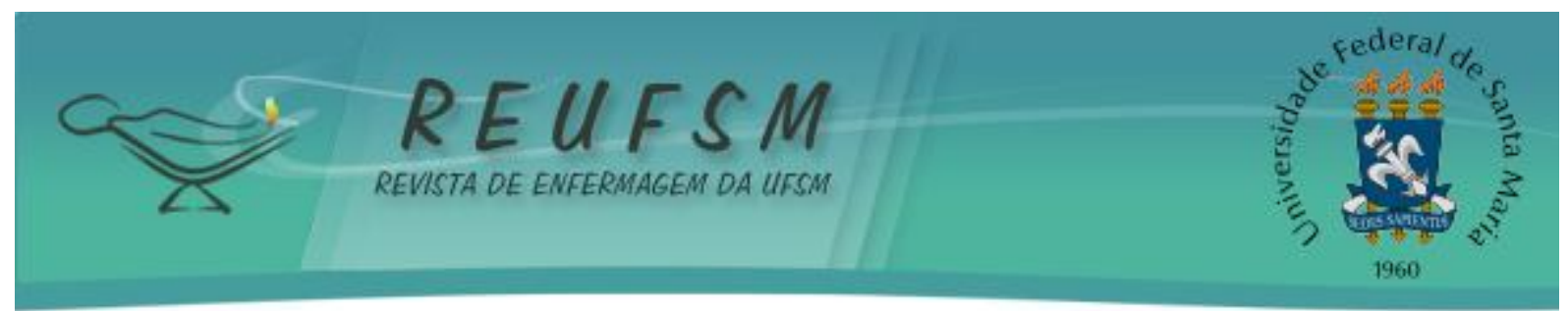

Quando se fala de SAE o que vem a minha mente são as facilidades do cuidar. Penso em métodos que fazem o atendimento de enfermagem serem mais eficazes, de modo a promover o bem-estar do paciente de modo mais completo possível. (Acadêmico 8)

Tecnologia utilizada pela enfermagem que facilita a prestação de serviços no âmbito da prática, o qual focaliza o paciente como o sujeito da assistência, tomando como método o histórico de enfermagem, diagnóstico e o processo de enfermagem. (Acadêmico 15)

Através da sistematização o enfermeiro planeja a sua ação e dessa forma o cuidado se torna efetivo. (Enfermeiro 11)

\section{O ensino da SAE e o cuidado prestado}

Nesta unidade os acadêmicos empregaram suas percepções ao ensino da SAE na FAENF/UFPA, onde as ideias principais levantadas foram "lacunas no ensino" e "divergências teóricas e práticas" segundo os textos abaixo:

O ensino da SAE deveria ser explorado com maior frequência em todos os campos de prática, já que, ela é imprescindível para a recuperação e conforto do paciente além de ser uma prática que somente se torna fácil quando é incorporada ao cotidiano do acadêmico de enfermagem. (Acadêmico 11)

Alguns docentes têm dificuldades no ensino da SAE e nem sequer a utilizam. Outros docentes tentam ensiná-la mostrando a importância científica desse processo no desenvolvimento das atividades exclusivas do enfermeiro. (Acadêmico 13)

Muitos professores falam teoricamente sobre a sistematização da enfermagem, porém, na prática ela não é muito aplicada. (Acadêmico 22)

Os enfermeiros por atuarem em uma instituição que é campo de prática dos discentes da FAENF/UFPA, acabaram por trazer informações sobre como o cuidado de enfermagem é visto pelos mesmos, o que intrinsicamente está ligado à formação profissional e a atividade assistencial, emanando, então, a ideia de que o "cuidado de enfermagem é fundamental".

A enfermagem é uma ciência e desenvolve ações como cuidar. É feita de forma planejada resultante da observação e análise de comportamento do ser humano individualmente. (Enfermeiro 15)

O cuidar da enfermagem é conhecer $e$ atender as necessidades do ser a ser cuidado, é dar oportunidade a ele de se cuidar e de desenvolver seu potencial. (Enfermeiro 8) 


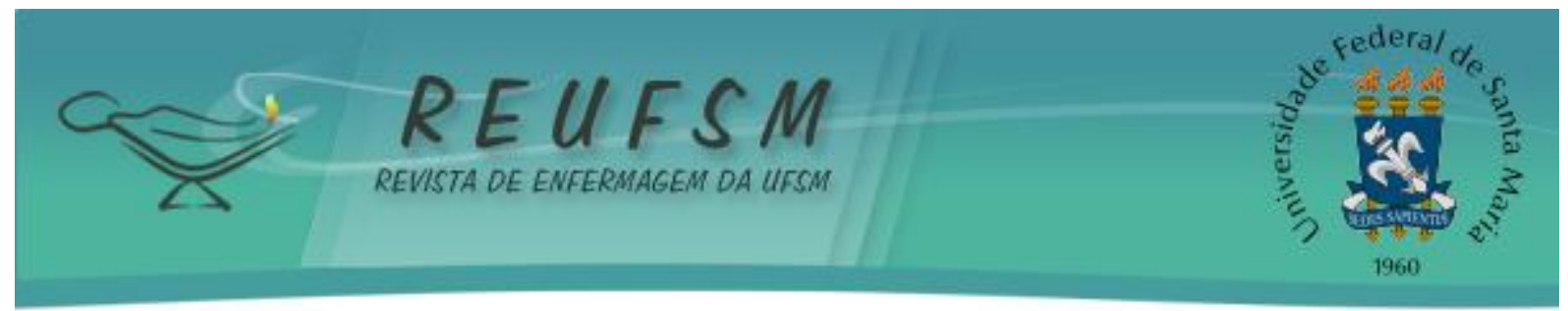

\section{A aplicabilidade da SAE}

A partir dos resultados percebe-se que para os acadêmicos de enfermagem a execução da SAE necessita de "experiência prática"; segundo este grupo é necessário ter "integração da equipe", ou o resultado será a "sobrecarga profissional”.

Durante as aulas práticas, tenho dificuldade quanto ao tempo para realizá-la cuidadosamente, já que precisarei realizar a investigação, exame físico detalhado, o diagnóstico de enfermagem (necessita de experiência, quando não se tem a NANDA ou CARPENITO em mãos), o planejamento, a implementação $e$ avaliação final. (Acadêmico 3)

As dificuldades encontram-se na própria execução da SAE pela falta de um processo de integração da equipe de enfermagem e da necessidade da equipe multiprofissional atuando junto. (Acadêmico 6)

As dificuldades enfrentadas são em relação ao exercício profissional e a sobrecarga de atividades, que envolvem registros, anotações, relatórios e comunicações, utilizando grande parte do tempo em atividades burocráticas e na busca de documentação das informações, possibilitando, assim, um afastamento da assistência centrada no paciente no desenvolvimento de atividades diárias essenciais como planos de cuidados, coleta de dados, planejamento, prescrição, coordenação e avaliação da assistência de enfermagem. (Acadêmico 5)

Os enfermeiros entrevistados também trouxeram ideias relativas à implementação da SAE como a "sobrecarga de trabalho", e o "reconhecimento dos demais profissionais de saúde", assim como, a SAE com fonte de "fortalecimento profissional" por meio da associação do conhecimento científico com a assistência de enfermagem.

Ainda na nossa realidade a implementação da SAE se torna difícil pelos inúmeros procedimentos burocráticos que temos que fazer $o$ que nos toma tempo que poderíamos estar utilizando para por em prática a SAE. (Enfermeiro 1)

A SAE não depende somente do interesse do profissional enfermeiro, mas também da colaboração dos componentes da equipe multiprofissional e esbarra também no interesse da instituição. (Enfermeiro 6)

É um processo que vem acompanhado de mudanças com ações inovadoras, buscando conhecimento e capacidade estratégicos para envolver os profissionais a partir de metodologias reflexivas. (Enfermeiro 2) 


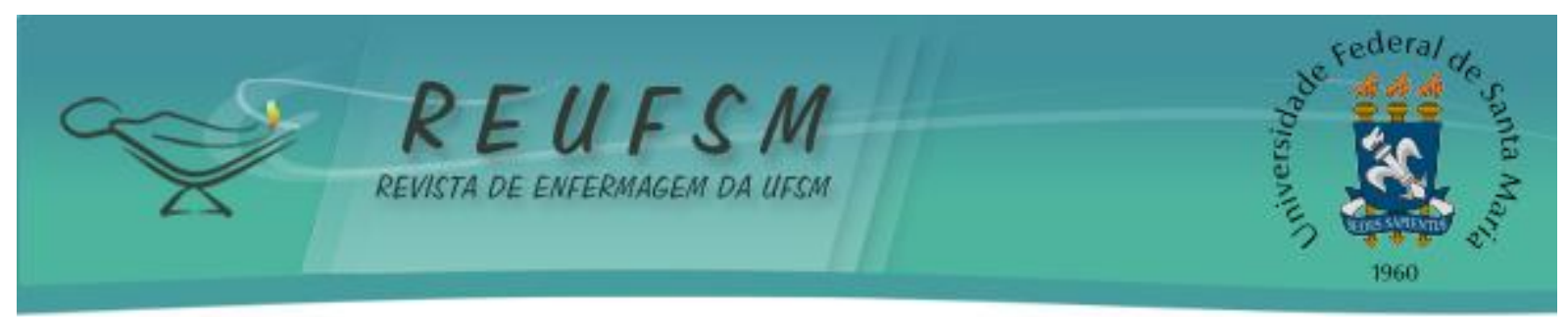

\section{DISCUSSÃO}

\section{As interpretações dos relatos}

Os grupos entrevistados, nos primeiros relatos, caracterizaram a SAE como um importante instrumento que permite a "autonomia do enfermeiro", o que possibilitou interpretar que os sujeitos sentem a necessidade de demonstrar, que a Enfermagem, têm competências e habilidades relevantes e inerentes a sua área de atuação, possuindo o mesmo respaldo científico que os demais profissionais da área da saúde. Portanto, as enfermeiras tem importante papel nas ações de saúde no fornecimento de atividades de baixo custo para os serviços de alta complexidade. ${ }^{7}$

Os acadêmicos de enfermagem compreendem a SAE sob um ponto de vista teórico, o que reflete a sua visão acadêmica, uma vez que os relatos constroem uma imagem metodológica do processo de enfermagem sem grande vivência com os resultados que a SAE pode proporcionar. Já os enfermeiros foram objetivos em seus depoimentos, trazendo a SAE como ferramenta para o aprimoramento da prática, propiciando o melhor cuidado, demonstrando então, uma visão completa do processo.

O desenvolvimento falho da SAE remonta um cenário demonstrando que ela é um instrumento metodológico, científico e filosófico no cuidado prestado, porém sua falha pode proporcionar um cuidar fragmentado, mecanizado, reducionista e despersonalizado. ${ }^{8}$ Acredita-se que um dos motivos da falha no desenvolvimento da SAE seja o ensino, que encaminha a esta dicotomia anteriormente citada, promovendo uma tradição na reprodução de lacunas educativas e, por vezes, descompromissado com a realidade.

Em relação ao ensino da SAE os alunos a descreveram em dois pontos distintos. 0 primeiro ponto trata-se da pouca associação entre teoria e prática, sendo fácil identificar por meio das colocações na primeira unidade, nas quais os acadêmicos de enfermagem entendem a importância do instrumento de cuidados e relatam quais as etapas do método. Entretanto, fazem pouca associação com a prática, justificado na segunda unidade, uma vez que mencionaram que nos campos práticos a SAE é pouco abordada. Segundo eles, trata-se do fato de alguns serviços de saúde não a desenvolverem como lhes é ensinado, ou simplesmente ela não é desenvolvida, o que na visão dos acadêmicos prejudica o seu aprendizado.

$O$ segundo ponto trata-se da dificuldade de docentes no desenvolvimento da SAE, com influências no aprendizado das disciplinas ministradas pelos mesmos.

0 docente em Enfermagem atua como formador de uma classe profissional, que deverá ter habilidades e competências na prática do cuidado, no entanto a cultura contemporânea pauta-se em atividades simplificadas e focadas em ações que obtenham resultados em curto prazo, e esta tendência influenciou diretamente o ensino, promovendo a dicotomia entre teoria e prática, baseado em uma formação pedagógica tradicional de um modelo tecnicista. ${ }^{9}$

As evidências levantadas no presente artigo já foram corroboradas na literatura, com o estudo ${ }^{10}$ que apontou para desafios a serem transpostos, como o desenvolvimento de estratégias que permitam os docentes, alunos e profissionais compreenderem a complexidade do cuidado e assim criar-se uma responsabilidade mútua pra a educação em Enfermagem, influenciando diretamente no processo de ensino aprendizagem da SAE.

Neste contexto, achou-se importante questionar os enfermeiros que participaram do segundo projeto, sobre o que é o cuidado de enfermagem, uma vez que a SAE está ligada diretamente as práticas assistenciais do enfermeiro.

O diferencial nos relatos dos enfermeiros foi a não descrição direta da SAE como um componente do cuidado de enfermagem. 


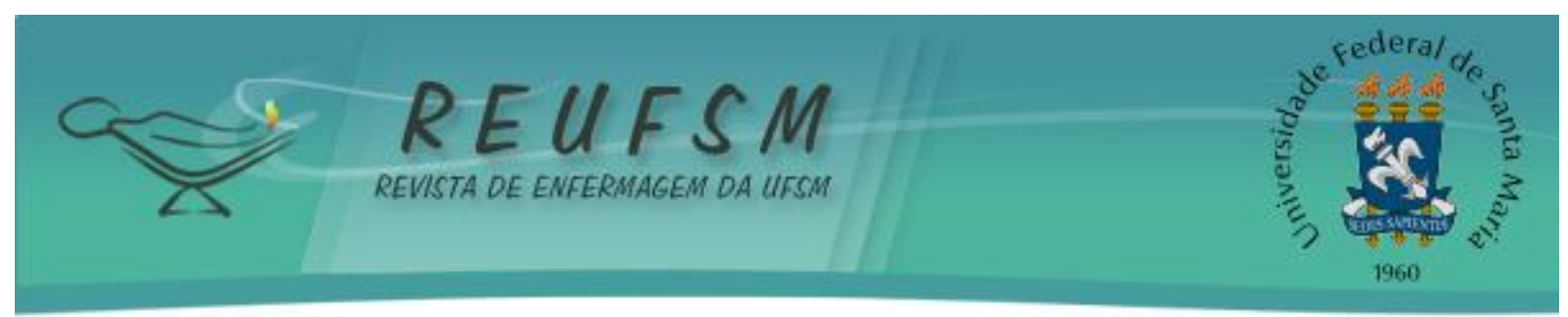

Os participantes do estudo caracterizaram a SAE como uma ação planejada, tendo como base a observação do ser humano, objetivando a regularização das necessidades básicas do indivíduo, ou seja, descreveram indiretamente uma ferramenta de cuidado; parte de sua operacionalização e que se consolida nas características da SAE, levando-se a inferir que o processo de enfermagem pode não ocorrer visivelmente aos olhos dos enfermeiros, mas está intrinsecamente imbricada nas ações de enfermagem.

Um estudo ${ }^{11}$ realizado em um hospital universitário corrobora os achados deste artigo, onde a SAE não era institucionalizada. Os enfermeiros ao descreverem o cuidado prestado apresentavam um método sistematizado que claramente pode ser correlacionado ao $\mathrm{PE}$, demonstrando que o raciocínio clínico da SAE é o essencial para o cuidado de Enfermagem, sendo o registro apenas uma comprovação legal das ações realizadas.

Apenas conhecer o PE não é suficiente ao enfermeiro. Ele necessita, além do saber teórico, ter a habilidade crítico-reflexiva que o permita criar um conjunto de valores, símbolos e ações a serem executadas, face ao julgamento das informações que foram coletadas, para que se possa atuar com qualidade no processo saúde/doença. ${ }^{12}$

A contraposição entre os relatos dos acadêmicos e dos enfermeiros levou-nos ao terceiro questionamento tratado na última categoria, onde se indagou aos dois grupos como ambos percebem a aplicabilidade da SAE em seus cotidianos.

Os acadêmicos de enfermagem relacionaram suas ideias sobre a aplicabilidade da SAE; com a necessidade de se ter experiência na execução, podendo desenvolvê-la de modo fidedigno aos seus pressupostos teóricos, sobretudo na execução do exame físico e sua associação com as etapas do processo de enfermagem, ou seja, apresentando uma visão mais tecnicista e mecanizada do PE, que foi herdada culturalmente da educação capitalista.

Para a mudança desta realidade é necessário não somente uma reformulação das práticas docentes, e das atividades assistenciais dos enfermeiros em busca de uma melhor formação, mas que exista um compromisso mútuo dos docentes e das instituições dos campos de prática, para que o acadêmico torne-se mais crítico frente às atividades que ele irá executar como profissional. ${ }^{13}$

A Enfermagem, por sua pretensão de cuidado integralizado, tende a atuar em equipe, e de modo geral é um excelente mediador entre os prestadores do cuidado e os usuários dos serviços de saúde. Infelizmente, a supremacia cultural do médico como detentor do conhecimento acaba por reduzir a visibilidade do enfermeiro perante a equipe de saúde, em muitos casos gerando o constrangimento. ${ }^{14}$

Também comum aos grupos, a visão da "sobrecarga de trabalho" para a Enfermagem. Para uns, seria consequência da dificuldade da gerência da assistência desde a graduação, o que segundo ambos os grupos, acaba por prejudicar a execução da SAE. Outro fator contribuinte para tal ideia refere-se a ausência de uma equipe multiprofissional trabalhando junto, o que impediria o acúmulo de funções e a sobrecarga do enfermeiro. Além de compreensão dos gestores ao empregarem a SAE no serviço, que os mesmos percebam os fatores que influenciam a aplicabilidade de qualidade ${ }^{13}$, uma vez que o excesso de atividades realizadas pelos enfermeiros possibilitam falhas em sua assistência, não refletindo apenas no PE, mas em outras atividades, tais como a administração de medicamentos, demonstrando a necessidade de reconfiguração das atividades dessa categoria. ${ }^{15}$

A cultura emerge da interação dos atores que estão agindo juntos para entender os eventos e procurar soluções, não sendo mais possível afirmar que a cultura impossibilita ao outro entender a nossa medicina, ou é um obstáculo a ser superado através de programas de educação em saúde. ${ }^{16}$ Portanto, é recomendado que os serviços de saúde utilizem da educação continuada como estratégia de implantação do PE e atualização dos profissionais, ressaltando a importância desta ferramenta para enfermagem, equipe 


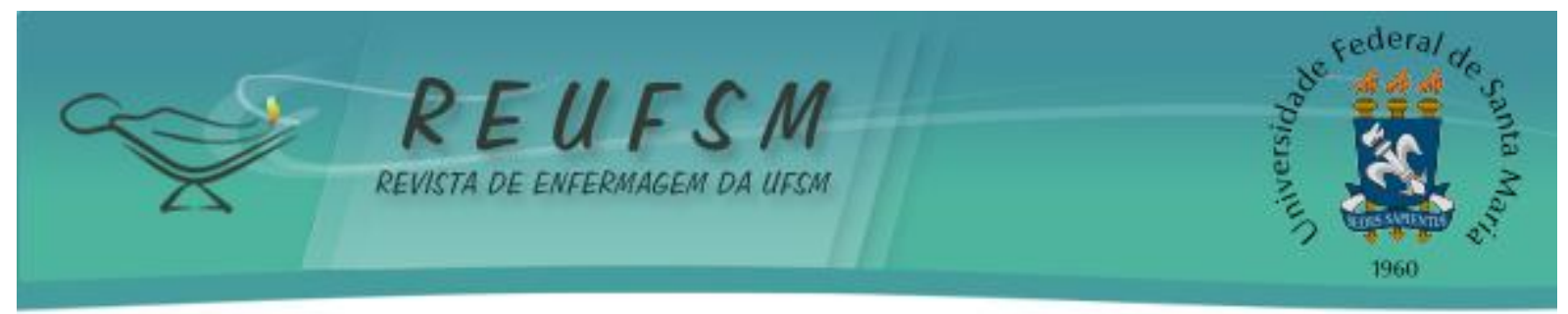

multiprofissional e para instituição de saúde. ${ }^{8}$ Desta forma fica claro que deve haver interação entre academia e os serviços de saúde. ${ }^{17}$

\section{CONCLUSÕES}

A SAE é um processo complexo que necessita ser desenvolvido com maior comprometimento entre os docentes e os enfermeiros dos campos de prática. Para isso os docentes devem ter percepção e habilidade em sua execução, permitindo a construção do saber dos acadêmicos centrados na importância e no desenvolvimento da SAE para a assistência de qualidade dos profissionais de enfermagem.

Os enfermeiros reconhecem a SAE como diferencial na prática do cuidado, porém, concomitantemente, queixam-se de sobrecarga de trabalho. 0 que por sua vez, com a união da categoria de enfermagem e a aproximação com a coordenação de enfermagem resultaria em intervenções para melhoria no setor hospitalar, com redistribuição e organização das tarefas, para que o enfermeiro consiga desenvolver as atividades de sua competência e assim promover um plano de cuidado adequado à realidade do paciente.

$O$ ensino e a aplicação do SAE, no contexto estudado, ainda não conseguem produzir resultados almejados, tais como a melhora no dimensionamento de enfermagem e união entre a equipe, o que facilitaria a execução da SAE na clínica. A participação efetiva dos docentes com domínio teórico e instrumental facilitaria a construção e aplicabilidade dos processos de enfermagem.

Consequentemente, os graduandos acabam não adquirindo as habilidades necessárias para o desenvolvimento do PE e reconhecem este instrumental como uma utopia do cuidado.

\section{REFERÊNCIAS}

1. Carvalho EC, Bachion MM. Processo de enfermagem e sistematização da assistência de enfermagem - intenção de uso por profissionais de enfermagem. Rev Eletrônica Enferm [Internet]. 2009 [acesso em 2014 fev 15];11(3):466. Disponível em: http://www.fen.ufg.br/revista/v11/n3/v11n3a01.htm.

2. Santos RB, Ramos KS. Sistematização da assistência de enfermagem em Centro Obstétrico. Rev Bras Enferm [Internet]. 2012 [acesso em 2012 jun 7];65(1):13-8. Disponível em: http://www.scielo.br/pdf/reben/v65n1/02.pdf.

3. Silva CC, Gelbcke FL, Meirelles BHS, Arruda C, Goulart S, Souza AlJ. O ensino da Sistematização da Assistência na perspectiva de professores e alunos. Rev Eletrônica Enferm [Internet]. 2011 [acesso em 2012 jun 8];13(2). Disponível em: http: //www.fen.ufg.br/revista/v13/n2/v13n2a03.htm.

4. Helman CG. Cultura, saúde e doença. $5^{\mathrm{a}}$ ed. Porto Alegre (RS): Artmed; 2008. 431 p.

5. Flick U. Introdução à pesquisa qualitativa. Porto Alegre (RS): Artmed, 2009.

6. Ministério da Saúde (BR). Conselho Nacional de Saúde. Comissão Nacional de Ética e Pesquisa (CONEP). Resolução $\mathrm{n}^{\circ}$ 466, de 12 de dezembro de 2012. Aprova diretrizes e normas regulamentadoras de pesquisas envolvendo seres humanos [Internet]. 2012 [acesso em 2012 ago 23]. Disponível em: http://conselho.saude.gov.br/comissao/eticapesq.htm.

7. Bryant R. The universality of nursing. International Nursing Review [Internet]. 2011 [acesso em 2012 out 18];58(1):1-2. Disponível em: http://onlinelibrary.wiley.com/doi/10.1111/j.1466-7657.2010.00875.x/abstract. 


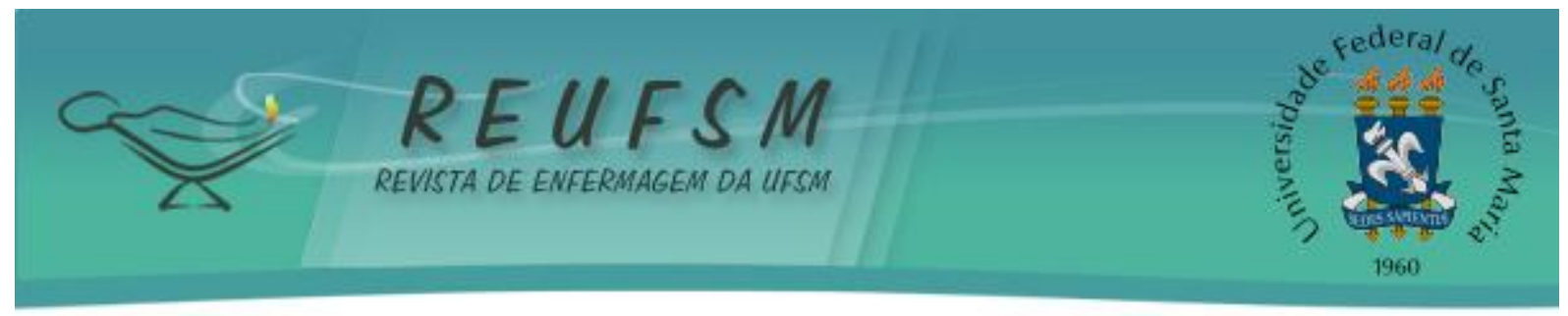

8. Menezes SRT, Priel MR, Pereira LL. Autonomia e vulnerabilidade do enfermeiro na prática da Sistematização da Assistência de Enfermagem. Rev Esc Enferm USP [Internet]. 2011 [acesso em 2012 ago 23];45(4):953-8. Disponível em: http://www.scielo.br/pdf/reeusp/v45n4/v45n4a23.pdf.

9. Silva MM, Moreira MC. Sistematização da assistência de enfermagem em cuidados paliativos na oncologia: visão dos enfermeiros. Acta Paul Enferm [Internet]. 2011 [acesso em 2012 set 1];24(2):172-8. Disponível em: http://www.scielo.br/pdf/ape/v24n2/03.pdf.

10. Silva AL, Camillo SO. A educação em enfermagem à luz do paradigma da complexidade. Rev Esc Enferm USP [Internet]. 2007 [acesso em 2012 set 1];41(3):403-10. Disponível em: http://www.scielo.br/pdf/reeusp/v41n3/09.pdf.

11. Torres E, Chistovam BP, Fuly PCS, Silvino ZR, Andrade M. Sistematização da assistência de enfermagem como ferramenta da gerência do cuidado: estudo de caso. Esc Anna Nery [Internet]. 2011 out-dez [acesso em 2012 set 1];15(4):730-6. Disponível em: http: //www.scielo.br/pdf/ean/v15n4/a11v15n4.pdf.

12. Malucelli A, Otemaier KR, Bonnet M, Cubas MR, Garcia TR. Sistema de informação para apoio à Sistematização da Assistência de Enfermagem. Rev Bras Enferm [Internet]. 2010 [acesso em 2012 set 12];63(4):629-36. Disponível em: http://www.scielo.br/pdf/reben/v63n4/20.pdf.

13. Silva EGC, Oliveira VC, Neves GBC, Guimarães TMR. O conhecimento do enfermeiro sobre a Sistematização da Assistência de Enfermagem: da teoria à prática. Rev Esc Enferm USP [Internet]. 2011 [acesso em 2012 set 12];45(6):1380-6. Disponível em: http://www.scielo.br/pdf/reeusp/v45n6/v45n6a15.pdf.

14. Pires D. A enfermagem enquanto disciplina, profissão e trabalho. Rev Bras Enferm [Internet]. 2009 [acesso em 2012 set 20];62(5):739-44. Disponível em: http://www.scielo.br/pdf/reben/v62n5/15.pdf.

15. Corbellini VL, Schilling MCL, Frantz SF, Godinho TG, Urbanetto JS. Eventos adversos relacionados a medicamentos: percepção de técnicos e auxiliares de enfermagem. Rev Bras Enferm [Internet]. 2011 [acesso em 2012 out 13];64(2):241-7. Disponível em: http://www.scielo.br/pdf/reben/v64n2/a04v64n2.pdf.

16. Langdon EJM. Cultura e os processos de saúde e doença. In: Jeola LS, Oliveira M, organizadores. Anais do Seminário Cultura, Saúde e Doença. Londrina: PNUD, Fundação Nacional de Saúde, Secretaria Municipal de Saúde; 2003.

17. Busanello J, Silva FM, Sehnem GD, Poll MA, Deus LML, Bohlke TS. Assistência de enfermagem a portadores de feridas: tecnologias de cuidado desenvolvidas na atenção primária. Rev Enferm UFSM [Internet]. 2013 [acesso em 2013 nov 20];3(1):175-84. Disponível em: http://cascavel.ufsm.br/revistas/ojs-2.2.2/index.php/reufsm/article/view/8532.

Data de recebimento: $29 / 10 / 2013$

Data de aceite: 03/04/2014

Contato com autor responsável: (016) 98822-7972.

Endereço postal: Rua Machado de Assis, 1034, Ribeirão Preto, São Paulo, Brasil. CEP: 14050-490.

E-mail: vandermonteiro@usp.br 